\title{
Virtual sector profiles for innovation sharing in process industry - Sector 01: Chemicals
}

\author{
Hélène Cervo ${ }^{1}$, Stéphane Bungener ${ }^{2}$, Elfie Méchaussie ${ }^{2}$, Ivan Kantor ${ }^{2}$, Brecht Zwae- \\ nepoel $^{3}$, François Maréchal ${ }^{2}$, and Greet Van Eetvelde ${ }^{1,3}$ \\ ${ }^{1}$ INEOS Europe, Lavéra, France \\ eposprojecteineos.com \\ ${ }^{2}$ EPFL Valais Wallis, Industrial Process and Energy Systems Engineering, Sion, Switzerland \\ ${ }^{3}$ Ghent University, Energy \& Cluster Management, Faculty of Engineering and Architecture,
}

Gent, Belgium

\begin{abstract}
Production data in process industry are proprietary to a company since they are key to the process design and technology expertise. However, data confidentiality restrains industry from sharing results and advancing developments in and across process sectors. Using virtual profiles that simulate the typical operating modes of a given process industry offers an elegant solution for a company to share information with the outside world. This paper proposes a generic methodology to create sector blueprints and applies it to the chemicals industry. It details the profile of a typical chemical site based on essential units and realistic data gathered from existing refineries and chemical plants.
\end{abstract}

Keywords: Virtual profile, sector blueprint, industrial data, chemicals sector, refineries, data confidentiality, industrial symbiosis, energy efficiency.

\section{Introduction}

Given the confidential nature of industry, handling process data typically implies nondisclosure agreements and - often exclusive - intellectual property rights granting protection to a company's background assets. More particularly, industrial data require protection because they are central to a company's plant or process design, crucial to their technology expertise and investments, essential in trade secrets, and most of all vital to feed innovation. Therefore, a good management of intellectual property is key to a company's competitiveness.

Securing and protecting data also has drawbacks, however, as industries are restrained from sharing best practices, advancing developments in and across sectors, transferring technology, building industry databases and benchmarks, feeding sector positions, creating synergies, etc. Additionally, it complicates the outreach of findings or results from research and innovation (R\&I) projects, even when funded through public grants. Horizon 2020 is a key example of such R\&I programme implementing the European Innovation Union. Through public-private partnerships such as SPIRE [1], companies are encouraged to participate in R\&I projects but the issue of data 
confidentiality remains the biggest hurdle for process industry to enter a project as full partner with a site-specific role.

Publications resulting from industrial research in collaboration with universities are intrinsically bound to a degree of public availability. Today, emerging concepts such as open source and open innovation, or industry-intrusive policies such as closing the loop of resources using circular economy principles, invigorate the need for answers on how to disclose information and facilitate communication across industry, academia and society. However, there is still a lack for publically available and reliable databases gathering information about industrial sectors operations and providing case studies to test and verify scientific developments. Furthermore, investigations with respect to data anonymisation techniques such as k-anonymisation, generalisation and Bucketisation, have been mainly carried in the field of individuals' data protection [2, 3] and have not been developed for industrial datasets.

The need to disclose industrial information and share project results has thus triggered the idea of creating a typical chemical site and a virtual sector profile. Anonymising industrial data as to enable communication between project partners and across process sectors demands for standardised methodologies. Therefore, typical industry sites and profiles per sector should be based on reality, generated through a recognised method and present representative data that are acceptable to all parties, industry and sector associations such as CEFIC for the chemical industry [4].

Building from the experience above and reinforced by the challenge of industrial symbiosis in the SPIRE project EPOS [5], this article presents a methodical and industry-proof approach to communicate on the chemicals sector by creating a virtual chemicals sector profile. This first section of the paper is followed by an analysis of the data needs and the structure of a virtual site in section 2. Section 3 shows the results of the methodology applied in the chemicals industry, followed by applications of such virtual profile in section 4. A final section summarises the conclusions of this work.

\section{$2 \quad$ Data sourcing}

As described in the introduction, typical industrial sites and profiles per sector should be based on reality and accepted by all parties, including sector associations. They should have identifiable units, use verifiable data and provide the means to present solutions matching the operating conditions of a real plant. A compromise should thus be found between the resolution and the relevance of data so as to select a representative set of data.

This section develops the methodology for building the profile of a typical chemical site based on essential units and realistic data gathered from existing refineries and chemicals plants. 


\subsection{Generic site layout}

The generation of a virtual chemical site profile implies the identification and characterisation of the major mass and energy flows across and within the site's boundaries, as well as their connections with the different building blocks composing the plant. Following a top-down approach, three levels of details to represent a plant layout can be defined: black box, site map and process unit representations.

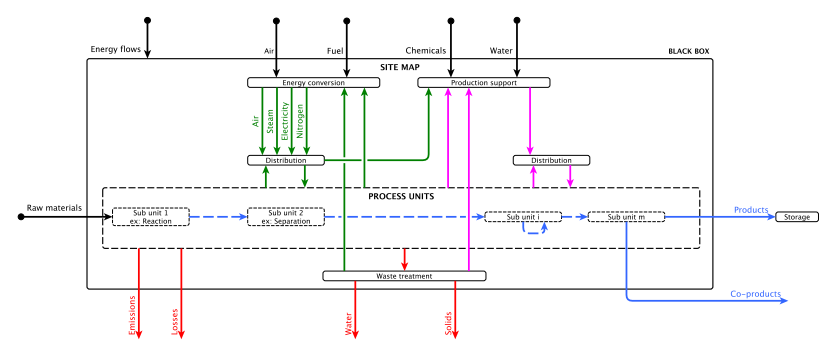

Fig. 1. Plant layout - representation levels [6]

Black box. The lowest level of detail is called the black box representation and is represented on Fig. 1 by the external black rectangle. Any cluster, site or plant can be characterised by its input and output flows of material and energy. This representation of a plant is the most general form and only gives insight into the main requirements or outputs. Each input and output flow can be used as an interface to connect with other sites or can be viewed as opportunities for internal usage as well. Nonetheless, this view on a site cannot provide details on opportunities for internal recycling or information about the real needs of the site in terms of material and energy unless it is assumed that the site is internally optimised and no further improvements are possible.

Site map. The next level of detail decomposes the site into its major sections, related to the production process, utility system or waste treatment and production support areas. This representation allows external observers to become familiar with the general layout of the plant and provides additional detail on the site operations. It enables understanding the main transformation steps onsite, from feedstock to product, including the major flows of materials and energy. Likewise, the utility demand and supply is represented per plant section. The boundaries of the sections are chosen according to the views of site engineers and the common understanding of the site.

This representation of an industrial site gives a good overview of the process units onsite, the supporting infrastructure and their interconnections. Numerous indications can be derived, such as the main energy consumers, the energy and mass balances per unit, the cold and hot utility distribution profiles or also the units' specific energy consumption. However, the process units' energy requirements (e.g. temperature levels and heat loads) are unknown, therefore it is not yet possible to generate a sufficiently detailed site profile using this second level of detail. 
Process units. For drawing a virtual profile, a third level of detail is required which enters the production process per unit. Via block flow diagrams the transformation steps per process unit are visualised. Each process unit groups a number of steps to transform material and energy inputs into useful outputs or final products.

The links between the process units are systematised and the logical flow of materials between the process units is captured. Again the units are defined from an engineering point of view by identifying the transformative operations and defining the boundaries for each process unit. While this is the most detailed site representation, the units remain common to chemical processes across the sector. As an example, steam-methane reforming (SMR) processes consist of similar transformation steps at most manufacturing sites.

With the deepest level of detail defined as the process unit, the next step is to select and allocate the necessary data to build the virtual profile of a chemical site.

\subsection{Simulated data and streams}

Energy flows. The energy vectors for the different representations identified in section 2.1 will vary according to the level of detail; for example, the black box level may show an input of natural gas while the subsequent levels show the conversion of gas into steam using a utility boiler. In order to build the virtual energy profile, data should first be collected with respect to the utility production and distribution (e.g. steam boiler and steam network). In a second step information about the utility consumption should be gathered. Mass and energy balances on the utility network should be closed at all points, considering losses inherent to large chemical sites.

While the utilities mass and energy flows are characterised by direct thermodynamic properties (temperature, pressure, low heating value, etc.), the utility consumption in process units is defined according to the real energy requirements at the interface between the process and the utility usage. This dual representation facilitates the definition of the utility requirements at the process unit level, reducing the complexity of data collection and enabling the identification of indirect/direct internal and external schemes for heat recovery (improve existing heat exchangers network, install external hot water circuit, etc.), heat pumping and cogeneration. Dedicated sources provide for more detailed explanations on data required to characterise process and utility streams [7].

Mass flows. Mass flows differ from energy flows due to the variable requirements of different processes and the plethora of possibilities for chemical conversion. Options for conversion of mass streams are almost unlimited.

For a plant represented by a black box, the interface with the rest of the world exists as the feedstock requirement and the production rate of the outputs which is often enough to understand the main purpose of the site and explore generic new business opportunities. However, mass requirements should be represented in a more detailed way since it could reveal material needs which do not exist at the higher levels, an example being a requirement for hydrogen in a process unit while the site imports only natural gas and generates hydrogen in a utility SMR unit. 
A further separation of mass flows is made to distinguish process needs from the transformation of inputs to outputs. Materials flowing in a network within a site can be defined at each point by their pressure, temperature, quality and flowrate such that the utility system is defined to provide these specifications (e.g. water network [8], hydrogen network [9]), they can be cascaded between process units or accessed at various points throughout the connected nodes. Material feed and product flows are defined similarly, though they are typically used in fewer points throughout the plant and are immediately transformed. These feedstock and product streams are the basis for the size of the process/plant in the virtual profile and are the basis by which the utility and production support streams are scaled.

Simulation method. Virtual profiles must be based on realistic and relevant data. However, companies are reluctant to disseminate specific data on their sites as they wish to retain the technical expertise and process specificities giving an advantage over their competitors. Thus, it is necessary to start from real plant data and apply a method that does not discern the specificities of the plant operations and process units. In the generation of a virtual chemical site profile, the anonymisation of data is coming from several aspects.

First of all, only the major mass and energy flows are included, even at the deepest level of detail. Sensitive specificities of processes linked to competitive advantages, mostly related to equipment design and operation, catalyst characteristics or processprocess integration (direct heat exchange between two or more process streams), are left out of the profile construction process and can therefore not be determined from the resulting representation.

The characterisation of the utility consumption within process units is made at the interface between process and utility streams, limiting greatly the disclosure of internal heat recovery schemes. For simplicity and generalisation purposes, the focus is being put on the most important heat requirements. A Pareto approach [10] can be followed to characterise $80 \%$ of the energy consumption, or a targeted approach can be preferred, focusing on the most significant energy use as defined by the ISO 50001 [11].

Finally, the construction of the profile implies the consideration of several process units, therefore all of them are merged together making it hard to precisely identify their specificities. No relevant information is lost in that process since the optimisation is made at the site level.

\section{$3 \quad$ Typical Chemical Sites}

This section introduces a virtual profile for the chemicals sector. The profile is obtained by applying the methodology described in section 2 and is further elaborated to produce a typical heat and power profile of a chemical site. The methodology can be extended to material streams or can focus on specific resources, waste streams, carbon emissions, etc. It is also applicable to various units giving the virtual profile some requisite flexibility (PUs can be added or removed if needed). 
The case study presented in this paper concerns an industrial site made up of a refinery (site R) and a chemical site (site C); it is referred to as a Typical Industrial Site (TIS) [6].

\subsection{Typical process units}

There are thousands of different chemical products, hence the profile that is built requires a helicopter view. It should be complete enough to give a general idea of the different conversion pathways that exist for a given chemical, while remaining sufficiently detailed to share information or discuss solutions across industries or with external actors.

The chemicals industry can be divided into two major subsectors, both either petroleum or bio-based [12]: refineries converting raw materials such as crude oil or biomass into fuels, chemicals and power and chemical sites which convert olefins, aromatics and natural gas into higher value products [13].

The refining and chemicals sectors are briefly described below as well as their main process units (PUs) and process flows.

Refineries. Fig. 2 shows the main PUs constituting a refinery as well as their main input and output flows. The conversion efficiency of a refinery can be as high as $93 \%$, meaning that $7 \%$ of the initial mass of crude oil is either used to provide energy to the PUs or evacuated as a waste product [14]. Waste streams are typically small as low value petrochemical fractions can be transformed into higher value products in PUs.

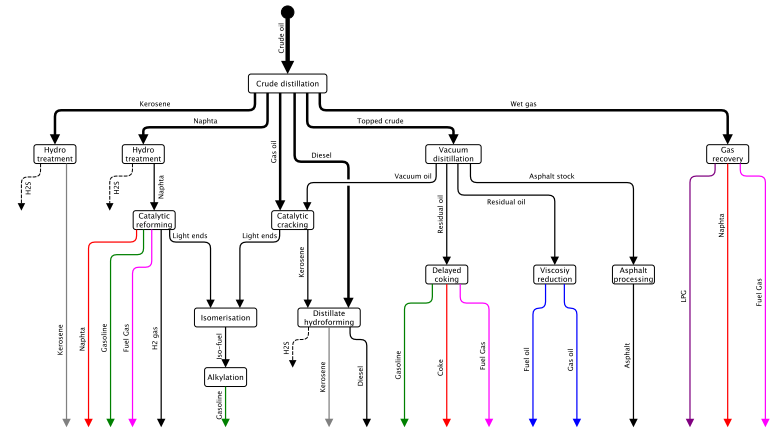

Fig. 2. Refinery main process units and mass flows [6]

Chemical sites. Fig. 3 illustrates the conversion pathways of oil fractions and methane into some of their end products. Each conversion of a feedstock into intermediary product implies significant energy input or output as well as many different types of units/processes: reactors, distillation columns, strippers, etc. The PUs selected for the creation of the profile are typical of a petrochemical site having a cracker unit. They are highlighted in red in Fig. 3. 


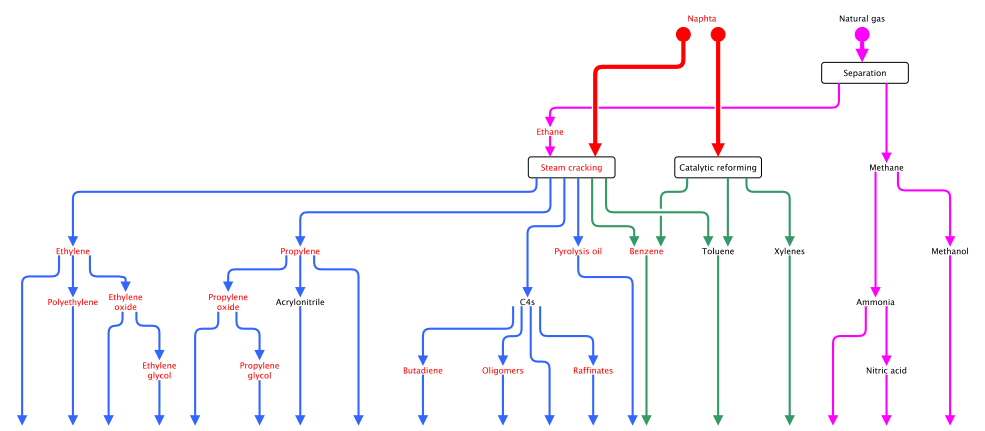

Fig. 3. Schematic of petrochemistry conversion pathways [6]

\subsection{Typical energy profiles}

Refineries and chemical sites are significant energy consumers. The utility system as well as production support units deliver streams such as demineralised water, steam and electricity which are essential for the PUs operations.

Heat profile. A Total Site Analysis (TSA) is performed in order to obtain the typical heat profile of the TIS. TSA [15] is a methodology where the utility system is integrated in the analysis and heat can indirectly be exchanged between processes using intermediate utility networks (ex. steam, hot water). In order to apply TSA, specific data are required to obtain the enthalpy profiles of both the PUs and utility system.

The systematic approach developed in [7] is followed and the required data set about the steam and cooling demand are integrated into the virtual profile. The steam demand corresponds to the amount of steam that must be produced by the utility network to supply PUs and utility demand with steam. It may include: boiler preheating, demineralised water degassing, tank tracing and steam turbine activation amongst others. Thermal losses and physical losses (leaks and condensation) also contribute towards the steam demand of an industrial site and should be included. The cooling demand is usually required after separation or to remove heat from exothermic reactions. The principal utilities used for cooling are air and water cooling.

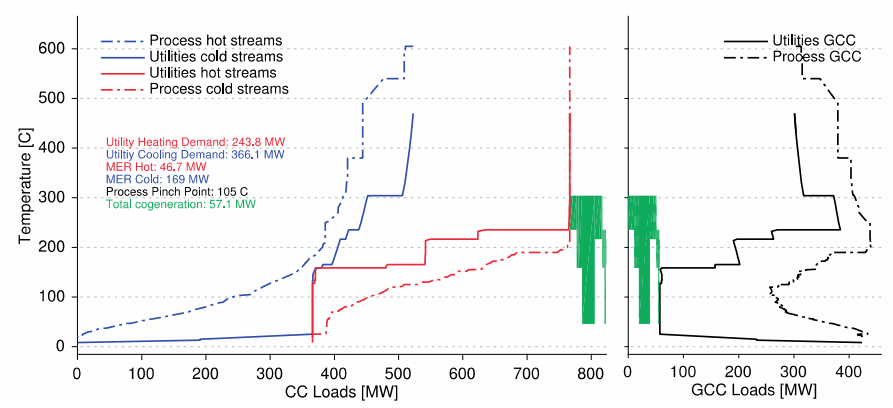

Fig. 4. CCs and GCCs of Typical Industrial Site for a given time period [6] 
The Composite Curves (CCs) and Grand Composite Curves (GCCs) of the TIS are shown in Fig. $\mathbf{4}$ for one specific point in time. However, the data are made available for multiple periods in order to account for the different site operating modes and the entire system variations. A specific methodology for clustering typical operation periods was developed in [16]. Since refineries and chemical sites are not necessarily coupled, CCs and GCCs can also be generated separately for site R and C, in order to analyse each of the site heat profile independently.

Electricity profile. Electricity is used as a resource in many production steps and it is critical to the safety of the system. A significant amount of the total power used by a site is often generated on-site (50-100\%). Two types of electricity production are typically found: internal combustion and turbines. The electrical consumption on a chemical site can be broken down into two main categories: pumping (50\%) and compressing (43\%). It is important to note that many processes in the chemicals industry have longer time constants than the electrical grid, resulting in a potential for demand response programmes [17].

The backbone of the TIS electricity system is the Medium Voltage (MV) rail system. This system is composed of redundant 3-phase rails with a voltage between $3 \mathrm{kV}$ and $36 \mathrm{kV}$. These rails are fed by High Voltage (HV) transformers, coupling the site to the external electricity grid with a working voltage between $70 \mathrm{kV}$ and $400 \mathrm{kV}$. The switchyard is the central node in the sites electricity system, which connects all units onsite. From the switchyard, electricity is fed into the site. Transformers feed distribution boards from this backbone. Large units might have a direct MV connection, e.g. large steam turbines. MV is used to distribute and transport the bulk of the electrical energy around the site. The MV system is also duplicated to provide redundancy and maintainability of the electrical system. Different paths can be selected from the switchyard to the distribution boards. In distribution substations onsite, the MV is finally transformed to low voltage (between $1 \mathrm{kV}$ and $400 \mathrm{~V}$ ). This is the working voltage of most small $(<100 \mathrm{~kW})$ machines and equipment.

\section{$4 \quad$ Virtual profile applications}

Once the concept of virtual sector profiles is adopted by industry and endorsed by the sector associations, the blueprints can be used to share best practices, screen new business potential across process industries or to challenge new policies. The virtual sector profiles are made to gain generic insights, produce a holistic site view, point to potential solutions or suggest possible ways forward for a plant or a sector.

Such an application can be illustrated via the use of the virtual energy profile [6]. First it shows the heating and cooling requirements of typical processes that can be found in the chemical industry while illustrating the way utilities deliver or remove the process energy. It thus enables to estimate the generic and internal optimisation potential of the system. On the other hand, this representation also highlights how much excess heat is available and transferable to potential external partners (district heating). Finally, it can also be used to display the opportunities for creating a 
common utility network with neighbouring sites in order to optimise the cogeneration and the energy integration. However, it should be noted that for each specific company or site case, an in-depth study must be completed to reach the required profundity for a grounded decision.

EPOS as an example. Projects such as EPOS [5], also prove the broad utilisation of virtual profiles. Prior to finding interactions and exchanges across process sectors, the sector blueprints are first used to create knowledge and a basic understanding of each process industry involved in the project (chemicals, steel, cement and minerals). Typical industrial sites are secondly used to map opportunities as well as barriers for cross-sectorial industrial symbiosis. In essence, the typical profiles of each sector are matched to explore industrial symbiosis (IS) potential without the need to disclose confidential data. Once an IS opportunity has been detected, the process is internalised and the involved industrial partners assess and validate the option using their own resources and site-specific data sets. When an opportunity results in a factual symbiosis, the economic, environmental, social and overall sustainability gains are calculated and extrapolated to the sector level. The process also works in reverse, starting from societal targets and pointing to new business opportunities across sectors by using the simulated sector profiles. Finally using virtual profiles also ensures a broad dissemination of the solutions detected through the entire EPOS project.

\section{Conclusion}

A generic methodology for building sector blueprints has been proposed and is applied to the chemicals sector. The virtual chemical site profile, based on essential units and realistic data gathered from existing refineries and chemical plants, provides a welcome solution for a company to share best practices and innovative solutions with the outside world without disclosing confidential information.

Data required for building a sector profile must be gathered at process unit level and that a trade-off should be made between the resolution and the relevance of information. The method is proven for building a typical heat and power profile of the chemicals sector and can be extended to mass flows covering resources and specific utilities. The profile is flexible since typical PUs can be added or removed while following the same methodology. At all times the virtual profile should be representative of the various operating modes of a real site in order to generate realistic solutions.

The use of virtual profiles opens up a range of applications. They enable sector benchmarks, substantiate sector positions, fuel prospection and impacts studies, etc. Similarly they grow the understanding of the chemicals sector, accelerate discussions between chemical companies and facilitate interactions across process sectors. Sectors blueprints also provide a welcome means to publish and present project results. They enable researchers to challenge scientific methodologies and tools and validate integrated solutions. Finally, they provide a common ground to policymakers for trying new strategies or, most of all, increasing the outreach of innovation projects funded through public grants. 


\section{Acknowledgment}

The EPOS project has received funding from the European Union's Horizon 2020 research and innovation programme under grant agreement No. 679386. This work was supported by the Swiss State Secretariat for Education, Research and Innovation (SERI) under contract number 15.0217. The opinions expressed and arguments employed herein do not necessarily reflect the official views of the Swiss Government.

\section{References}

1. SPIRE, https://www.spire2030.eu/

2. N. V. Mogre, G. Agarwal and P. Patil: A review on data anonymization technique for Data publishing. International Journal of Engineering Research \& Technology (IJERT), vol. 1, no. $10(2012)$

3. G. Cormode and D. Srivastava: Anonymized Data: Generation, Models, Usage. In: Proceedings of the ACM SIGMOD International Conference on Management of Data (SIGMOD 2009), Providence (2009)

4. CEFIC - The European Chemical Industry Council, http://www. cefic.org/

5. EPOS - Symbiosis in Industry, https : / /www. spire2030.eu/epos

6. L. G. S. Bungener, Energy Efficiency and Integration in the Refining and Petrochemical Industries. PhD thesis. Ecole Polytechnique Fédérale de Lausanne (2016)

7. E. Méchaussie, S. Bungener, F. Maréchal and G. Van Eetvelde: Methodology for streams definition and graphical representation in Total Site Analysis. In: 29th International Conference on Efficiency, Cost, Optimization, Simulation and Environmental Impact of Energy Systems (ECOS 2016), Portorož (2016)

8. Natural Resources Canada, Pinch Analysis: For the Efficient Use of Energy, Water \& Hydrogen (2003)

9. N. Hallale and F. Liu: Refinery hydrogen management for clean fuels production. Advances in Environmental Research, vol. 6, no. 1, pp. 81-98 (2001)

10. J. Juran: Pareto, Lorenz, Cournot, Bernoulli, Juran and Others. Industrial Quality control, p. $25(1960)$

11. ISO 50001:2011, Energy management systems - Requirements with guidance for use

12. E. De Jong, A. Higson, P. Walsh and M. Wellisch: Bio-based Chemicals: value added products from biorefineries. IEA Bioenergy Task 42 Biorefinery (2010)

13. Petrochemistry - Association of Petrochemicals producers in Europe, http://www.petrochemistry.eu/flowchart.html

14. M. Wang, H. Lee and J. Molburg: Allocation of Energy Use in Petroleum Refineries to Petroleum Products Implications for Life-Cycle Energy Use and Emission Inventory of Petroleum Transportation Fuels. The International Journal of Life Cycle Assessment, vol. 9 (2003)

15. V. R. Dhole and B. Linnhoff: Total Site Targets for Fuel, Co-Generation, Emissions and Cooling. Computers and Chemicals Engineering, pp. 101-109 (1993)

16. L. G. S. Bungener, G. Van Eetvelde and F. Maréchal: A Methodology for Creating Sequential Multi-Period Base-Case Scenarios for Large Data Sets. Chemical Engineering Transactions, vol. 35, pp. 1231-1236 (2013)

17. I. Mohd Noor, N. F. Thornhill, H. Fretheim and E. Thorud: Quantifying the demand-side response capability of industrial plants to participate in power system frequency control schemes. In: PowerTech, 2015 IEEE Eindhoven, Eindhoven, (2015) 